\title{
A Chaos Time Series Prediction Method of Generalized Phase Space
}

\author{
Yu-min Pan ${ }^{* 1, a}$, Yong-hong Deng ${ }^{1, b}$, Li-feng Wu ${ }^{1}$, Quan-zhu Zhang ${ }^{1, \mathrm{c}}$ \\ ${ }^{1}$ Information and Control Technology Institute of North China Institute of Science \& Technology Beijing, 101601, China/ \\ College of Information Engineering, Capital Normal University, Beijing 100048, China \\ ${ }^{* a}$ pym_001@ncist.edu.cn; bdyh1081@163.com; ${ }^{\mathrm{c}}$ zhangqz608@ ncist.edu.cn
}

\begin{abstract}
Aiming at the limitation of the regression prediction method for mine gas emission, a chaotic time series prediction method for reconstruction of generalized phase space is proposed in this paper. This method is used to construct phase spaces for time series with unobvious chaotic characteristics and fit equivalent chaotic attractors by RBF and BP network in order for prediction researches by calculating saturated embedding dimensions and designing main neural network parameters. A prediction method for stabilizing the neural network prediction result is brought forward and wavelet denoising is made for original signals to improve the prediction precision. This method can effectively overcome the deficiency of record data types, with stronger practicability, then the simulation experiment on Mackey-Glass time series and actual mine gas emission has proved the feasibility and effectiveness of this method.
\end{abstract}

Keywords-Chaotic; Phase space reconstruction; RBF; Distribution coefficient; BP; Gas emission; Wavelet denoising

\section{INTRODUCTION}

Multiple regression prediction method is a major method for the neural network prediction of gas emission [Hong-qing ZHU et al., 2005; Chuan-qu ZHU, 1999; Song GU et al., 2009] This method employs multiple impact factors as neural network input and gas emission as network output and adopts the sample set training neural network to construct a prediction model and utilizes the neural network extrapolation ability (also generalization ability) to achieve the predicted value of detection sample. The existing main problem of this method is to present the numerical record of all impact factors at the next moment when predicting the gas emission at the next moment, so as to carry out the trend recursion prediction. For most mines, especially small and medium-sized coal mines, however, it is difficult to provide 9 impact factor data similar to those in literature (Song GU et al., 2009), but few data are recorded, such as gas emission or gas content, wind rate or mine pressure, etc. Accordingly, how to accurately predict the gas emission under the less data information is now a realistic research theme for mine safety early-warning.

Comparatively, time series prediction method only needs a one-dimension historical data record to predict the future trend, which is more suitable for actual mine conditions. But the time series prediction method is generally lower than the precision of the regression prediction method, sometimes with bad effect and greater prediction difficulty.
The chaotic time series prediction method based on phase space is used to connect time series and multiple regression prediction method so that the time series prediction is transformed into equivalent multiple regression prediction. As Takens'opinion, the original system phase space can be reconstructed according to the time series of a variable and the original dynamic system can be recovered from any series (Min HAN, 2007). Takens has proved that a proper embedding dimension can be found out, namely keep a diffeomorphism with the original power system on the trajectory in the reconstructed space, and Packard et al recommended using delay coordinates of some variables in the original system to reconstruct the phase space (Xiang-cheng JIANG, 2011).

At present, there are few researches on prediction of the gas emission by the chaotic time series prediction method. Based on the improvement of Lyapunov exponential, the gas emission time series is predicted in literature (Wen-biao HUANG and Shi-liang SHI, 2009) to achieve a higher accuracy; the chaotic time series is employs to construct the phase space and the neural network is used to predict the gas emission rate in literature (Wei-wei HUANG et al. 2005); A false nearest neighbor is used for the gas concentration time series to confirm the embedding dimension, a mutual information method is used to confirm the time delay and a weighted onerank local region method is adopted to establish a gas concentration prediction model in literature (Jian CHENG et al., 2008); based on the small-data method to determine the time series chaotic characteristics and combine the wavelet multiresolution characteristics with $\mathrm{RBF}$ to improve the gas content prediction accuracy, etc.

The chaotic time series prediction method above is based on a premise, namely the gas emission or gas concentration time series must have the chaotic characteristics, but this method is not applied to any time series which has no chaotic characteristics. Mine gas data have a worse regularity mostly but the average cycle approximates to the data length, so it is difficult to differentiate the chaotic characteristics. Currently, the selection of delay time and embedding dimension is subjective to different extent during reconstruction of phase space, information verification is firstly performed for a phase space which has no time series, so it is a lack of a definite objective to determine the reconstruction effect of the phase space, and there has not been any universal effective method 
available up to now, so that this kind of prediction method is a little difficult during actual application (Li GAO et al., 2008).

A chaotic time series prediction method for generalized phase space is proposed in this paper to predict the time series with fuzzy chaotic characteristics. This method employs an exhaustion method to determine the saturated embedding dimension, delay time and main neural network parameters, so that the prediction model has only the advantage of multiple regression prediction but also the characteristic that time series prediction method requires less time series data sequences.

\section{CAlCulation OF PHASE SPACE PARAMETERS}

The time series data of a complex system is a complex time-space evolution of nonlinear dynamic system, the dynamic time data series of its physical and mechanical responses must contain traces and characteristic information of the dynamic evolution process (Chun-bo XIU and Meng XU, 2010). In accordance with Kakens theorem, the dynamic behavior of system evolution can be expressed without singularity by the evolution track of points in the proper dimension phase space composed of one-dimension observation series and proper time delay values (Kai WANG and Qi-xiang YU, 2005). Usually, delay time is expressed by $\tau, \mathrm{m}$ indicates statured embedding dimension, if the original series is $x(t), t=0,1,2, \cdots, n$, the phase space is expressed as follows:

$$
\begin{gathered}
p(t)=[x(t), x(t+\tau), \cdots, x(t+(m-1) \tau)] \\
t=0,1,2, \cdots, n-(m-1) \tau
\end{gathered}
$$

In the formula, $N=n-(m-1) \tau$ is the total of phase points in the reconstructed phase space, which indicates the effective length of vector series, namely vector length of phase space after reconstruction. The reconstruction of state space intends to recover chaotic factors in the high-dimensional space and research the evolution law, which is the theoretical basis for researches on the prediction of chaotic time series.

\section{A. Chaotic Time Series Prediction Model Based on RBF Network}

M-dimensional variables of phase space after reconstruction are taken as RBF neural network input to fit chaotic attractors. The prediction model is as shown in Fig.1.

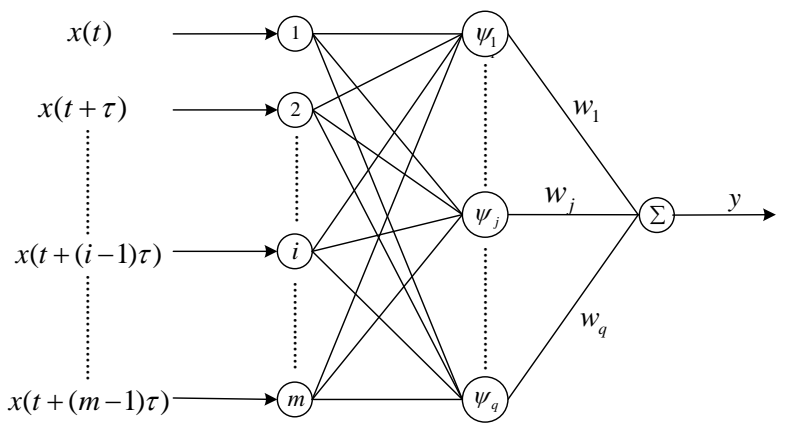

Fig. 1. Use prediction model based on phase space and RBF network
In Fig.1, the network structure is $m-q-1$, $\mathrm{m}$ indicates saturated embedding dimension and $\mathrm{q}$ indicates number of hidden-layer neurons, the radial basis function is expressed as follows:

$$
\varphi_{j}\left(\left\|x-c_{j}\right\|\right)=\exp \left(-\frac{\left\|x-c_{j}\right\|^{2}}{2 b_{j}^{2}}\right), j=1,2, \ldots, q
$$

In Fig.1, RBF network input layer/hidden layer connection weight is $1, w_{j}$ indicates hidden layer/output layer connection weight, this model realizes a single-step prediction. Given the hidden layer output matrix is $\boldsymbol{\Phi}=\left(\varphi_{1}, \cdots, \varphi_{q}\right)$, the network output is $y=\boldsymbol{\Phi} \boldsymbol{W}$. In the above prediction model, the determination of $\tau$ and $\mathrm{m}$ is a key for this prediction model. In the reconstructed phase space, the selection of delay time and embedding dimension is extremely significant.

\section{B. Non-uniqueness of Determination Methods of arameters $\tau$ and $m$ in the Phase Space}

In Takens theorem, for the ideal one-dimension time series for infinite length and no noise, delay time and embedding dimension can be valued randomly, but the time series has infinite length and noise available during actual application. $\tau$ and $\mathrm{m}$ are selected by the following two methods:

- Separate solution, for example, the delay time $\tau$ is determined by self-correlation method, mutual information method and so on; $\mathrm{m}$ is determined by Cao-style method, G-P method, false nearest neighbor, etc.

- $\tau$ and $\mathrm{m}$ are solved unitedly by C-C method, tmime window method proposed by Broomhead and King and so on, all of which specifies the integral product relation between $\tau$ and $\mathrm{m}$ (Jin-hu LV et al., 2005).

- Ataei et al specified different prediction errors are selected for some typical chaotic systems under different delay time and embedding dimension conditions in literature (Chun-Tao ZHANG et al., 2010).

Therefore, phase space parameters confirmed by traditional method are not necessarily the optimal and can't soundly keep the integral characteristics of the original power system(ATAEI M et al., 2004).

\section{Simulation Examples}

Mackey- Glass time series is a typical chaotic time series and educed by delay differential equation as follows:

$$
\frac{d x(t)}{d t}=\frac{\alpha x(t-\tau)}{1+x^{10}(t-\tau)}-\beta x(t)
$$

Given $\alpha=0.2, \beta=0.2$, when $\tau>17$, the time series shows chaotic characteristics, the larger $\tau$ value is, the higher the chaotic degree becomes. Fig. 2 indicates chaotic time series 
when $\tau=18$. Four-order Runge-Kutta method is applied to seek for numerical solutions in formula (3) and educe the chaotic time series, $\mathrm{x}$ is taken as observation variable to generate 10000 data, removing first 5000 transient data but taking the subsequent 1000 data as shown in Fig. 2, of which 600 data are taken as training set and 400 data are used to detect the properties of the model.

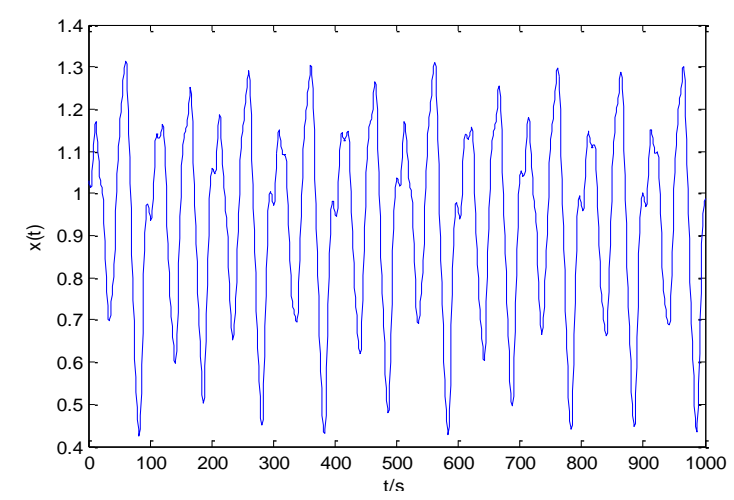

Fig. 2. Original data of macke-glassy

After calculation, the average cycle of the data is $\mathrm{P}=50$, the mutual information method is used to determine the delay time as $\tau=7$, Cao-style method is used to educe the saturated embedding dimension $\mathrm{m}=3$; if $\mathrm{C}-\mathrm{C}$ method is adopted, $\tau=10$, $\mathrm{m}=8$. G-P method is employed to determine the fractal dimension which approximates to 2.12 , in the case of $m \geq 2 d+1$, then $\mathrm{m}=6$.

(1) When $\tau=7, \mathrm{~m}=3$, RBF neural network is adopted to fit chaotic attractors, if RBF network spread coefficient is 4 , the absolute average prediction precision is $0.3732 \%$, the maximum absolute average prediction precision is $1.8393 \%$ and the maximum error is 0.0214 .

(2) When $\tau=10, \mathrm{~m}=8$, spread $=4$, the absolute average prediction precision is $0.0039 \%$, the maximum absolute average prediction precision is $0.0397 \%$ and the maximum error is 0.0003 .

(3) When $\tau=10, \mathrm{~m}=6$, spread $=4$, the absolute average prediction precision is $0.0027 \%$, the maximum absolute average prediction precision is $0.0158 \%$ and the maximum error is 0.00016 .

The calculations above show that different analyses result in inconsistent prediction results, because different methods can't deem prediction results as bases to be considered. Actually, there is an optimal prediction precision available, which can be searched by introducing an optimization algorithm.

\section{III. $\tau, \boldsymbol{M}$ AND $\boldsymbol{R} \boldsymbol{B} \boldsymbol{F}$ NETWORK PARAMETERS}

In RBF neural network, spread indicates distribution coefficient of radial basis function, a rational selection of spread is important and its value should be large enough, so that the radial base neurons are able to respond the sections covered by input vectors. RBF neural network and other neural network predict different points, which should be the uniqueness of the prediction result, namely repeatability, and the distribution coefficient is the unique parameter controlling the RBF network properties. RBF network created by Newrbe function order can automatically select the number of hiddenlayer neutrons without training process and accelerate the network speed of small-scale data.

The evolution of attractors in the phase space is fit by BP, RBF or wavelet neural network to reveal the motion laws of the system. As for the prediction model in Fig. 1, RBF network is adopted to fit chaotic attractors, $\tau$, $m$ and RBF distribution coefficient spread can be educed by exhaustion method as follows: $\tau=4, \mathrm{~m}=6$, spread=1(Chang-sheng XIANG et al., 2011). The phase space is reconstructed and RBF network parameters are set according to these parameters. Simulation test is made in MATLAB R2009a environment, and data after normalization are input in the network training for prediction. The prediction result is as shown in Fig. 3.

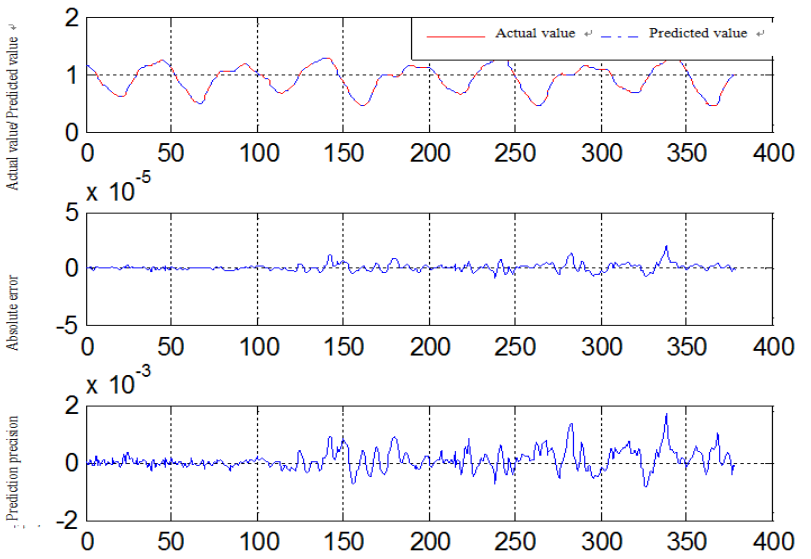

Fig. 3. Predivtion result of macke-glassy time series

In Fig.3. the absolute average prediction precision is $0.000259 \%$, the maximum absolute average prediction precision is $0.0017 \%$, the maximum error is 0.00002068 and the training \& prediction time totals $1.1164 \mathrm{~s}$. The prediction result achieves the comparatively high precision, which is much better than the effect of parameter prediction determined by chaotic theory.

The experimental result above shows that the prediction of Lorenz time series by saturated embedding dimension and delay time solved by chaotic theory is not always the optimal, there must be an optimal value available, which can be educed by searching.

\section{GENERALIZED PHASE SPACE PARAMETERS AND PREDICTION}

For chaotic time series, the system is determined whether it has chaotic characteristics by constructing the phase space and calculating chaotic parameters such as fractal dimension, Lyapunov exponential or Kolmogorov entropy etc. During the actual construction, however, there are still many time series 
which have not chaotic characteristics, or chaotic characteristics are not obvious, when the chaotic theory or prediction method will be inapplicable any longer, such as hydrological time series, traffic flow, mine gas emission or gas content time series, etc. The prediction method for chaotic time series is a nonlinear system time series prediction method essentially, it does not require that time series is strict and chaotic (Yong YOU et al., 2002). Hence, the chaotic time series prediction method can be popularized. As for non-steady time series, its saturated embedding dimension and delay factor can be generally solved when chaotic characteristics are not considered.

The time series of gas emission on the mine working face is usually a sequence of data with worse regularity. Gas emission is a main source of any gas accident and the most important coal mine hazard during safe production, and it directly impacts the mine construction, exploration of coalbed gas, mine safety, economic and technical indexes (Wen-zhong JIANG et al., 2008). The gas emission shows an obvious uncertainty due to influences from coal yield, exploration strength, exploration depth expansion, etc.

Because the coal seam gas content is affected by many geologic factors and gas is distributed uniformly in the coal seams, the time series of gas emission features nonlinearity, randomness, fuzziness and so on, and these characteristics are more obvious with the increase of gas exploration depth, so that the traditional prediction method suffers from great difficulties, applicable data for most mines are deficient and the effectiveness of multiple regression prediction method reduces. The high-dimensional phase space prediction research on establishment of gas by time series prediction method is thereby significant.

\section{A. Prediction Example}

The actual data, which is acquired from the gas emission from 8463 working face of Yangdong Mine of Jizhong Energy Group, is tested as shown in Figure 4. The data, which are acquired from the site from April 1, 2011 to May 7, 2011, include 110 in total.

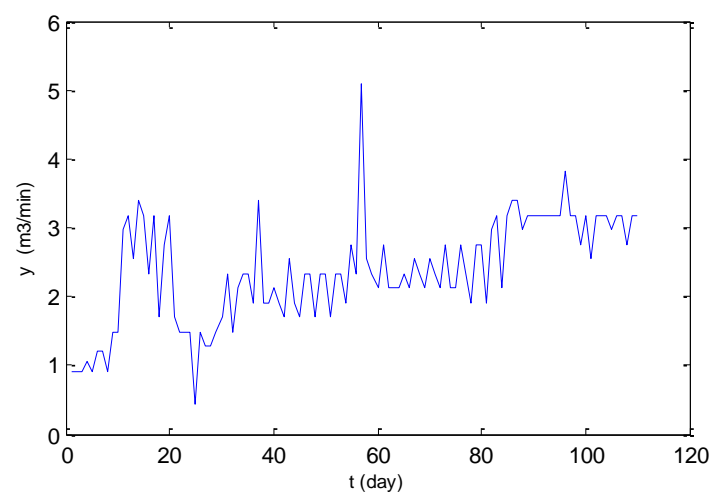

Fig. 4. Original data of the gas emission

On the basis of chaotic characteristic analyses, the average cycle of this series is $\mathrm{P}=109$, mutual information method is adopted to educe the delay time $\tau=1$, Cao-style method is used to determine the saturated embedding dimension $m=10$; if $\mathrm{C}-\mathrm{C}$ method is adopted, we can educe $\tau=1, m=20$. Because the average cycle approximates to the data length, it is difficultly educed by Lyapunov exponential and chaotic characteristics are not determined easily; if the fractal dimension method is adopted as shown in Figure 5, the fractal dimension $\mathrm{d}$ fails to tend to a stable value as shown in Fig. 5 (b), so the chaotic characteristics are not identified.

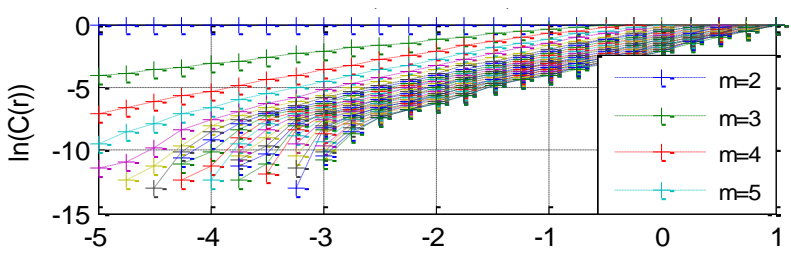

(a) $\ln (r)$

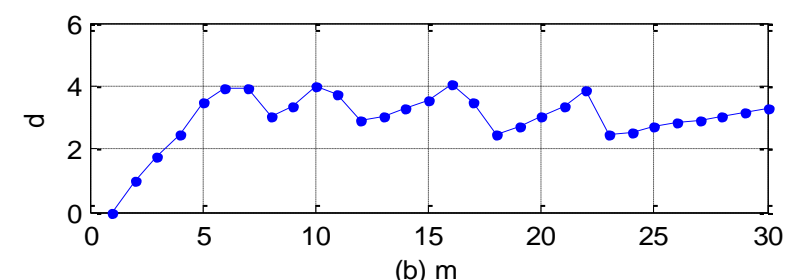

Fig. 5. Embedding dimension by g-p method

Due to smaller data length in this paper, the phase space is reconstructed according to $\tau=1, m=10$. The first 80 data are used for training network and the last 30 data for checking model. The experiment shows that the effect is bad if RBF is used for prediction, so RBF network is inapplicable to this data $\mathrm{RBF}$ is a local network, and it is difficult to extract its characteristics for data with worse regularity, so the prediction is performed by BP or wavelet neural network and so on. In accordance with the experimental effect, 15 neutrons are taken from BP network hidden layer, the network structure is 10-15-1 and the training precision is set as 0.009 , but the prediction effect is bad through experiment. Different from RBF network kit, the prediction result of $\mathrm{BP}$ network kit is not unique; especially for the data as shown in Figure 4, multiple predictions show a higher dispersancy. If the prediction result is an unrepeatable random number, it is difficult to quantitatively determine its prediction performance.

The initialization process of neural network parameters is very important for the convergence and convergence rate of the subsequent network learning; if the initial weight is selected well, the convergence speed is accelerated greatly; if the initial weight is set improperly, the learning frequency will be increased greatly and even no convergence is available (Xuezhi ZHAO et al., 2003).

A new BP network parameter initialization method is adopted in this paper. Before creating BP network, rand ('state', Q) order is executed to achieve an extremely stable prediction result by adjusting and optimizing $Q$ value. In MATLAB software, rand ('state', Q) order indicates the resetting of 
generator to the original state, state indicates the state of random number. Because random numbers are not the same each time, this function is used to generate identical random numbers in order to educe a result with the same as former some state.

Rand ('state', Q) order is used for experiment, which can make the network prediction result is repeatable and realize the comparison of different networks under the same conditions, which is extremely important for prediction.

We can educe $\tau=4, m=5, \mathrm{Q}=14$ through calculation. BP network structure is $5-15-1$, an equivalent phase space is reconstructed according to calculated delay factor, saturated embedding dimension, etc., and BP network is used to fit equivalent chaotic attractors. As for data as shown in Figure 3, in order to extract its characteristics, the first 80 data are used for training network, and the last 30 data for checking model. The prediction result is as shown in Table 1 .

TABLE I. BP NETWORK PREDICTION RESULT IN THE CASE OF Q=14

\begin{tabular}{|c|c|c|c|c|}
\hline No. & $\begin{array}{l}\text { Actual time } \\
\left(m^{3} / \min \right)\end{array}$ & $\begin{array}{c}\text { Predicted } \\
\text { value } \\
\left(m^{3} / m i n\right)\end{array}$ & $\begin{array}{c}\text { Error } \\
\left(\mathrm{m}^{3} / \mathrm{min}\right)\end{array}$ & $\begin{array}{c}\text { Precision } \\
(\%)\end{array}$ \\
\hline 1 & 3.180 & 3.3462 & 0.1662 & 4.9668 \\
\hline 2 & 2.756 & 3.4263 & 0.6703 & 19.5627 \\
\hline 3 & 3.180 & 3.1973 & 0.0173 & 0.5402 \\
\hline 4 & 2.544 & 3.5543 & 1.0103 & 28.4240 \\
\hline 5 & 3.180 & 3.0207 & -0.1593 & -5.2726 \\
\hline 6 & 3.180 & 3.2451 & 0.0651 & 2.0047 \\
\hline 7 & 3.180 & 3.1284 & -0.0516 & -1.6488 \\
\hline 8 & 2.968 & 3.4678 & 0.4998 & 14.4114 \\
\hline 9 & 3.180 & 3.0512 & -0.1288 & -4.2228 \\
\hline 10 & 3.180 & 3.3433 & 0.1633 & 4.8846 \\
\hline 11 & 2.756 & 3.1787 & 0.4227 & 13.2975 \\
\hline 12 & 3.180 & 3.1165 & -0.0635 & -2.0380 \\
\hline 13 & 3.180 & 3.0020 & -0.1780 & -5.9288 \\
\hline $\begin{array}{l}\text { Aver } \\
\text {-age }\end{array}$ & --- & --- & 0.2766 & 8.2464 \\
\hline
\end{tabular}

As shown in Table 1, the absolute average prediction value is $8.2464 \%<10 \%$, which indicates the prediction of gas emission for actual production.

\section{B. Prediction of Conventional BP Network and Wavelet Neural Network}

1) Adoption of BP neural network: the first three-day data in the original data are taken as neural network inputs and the data in the fourth day as network outputs to successively recur and establish training data and predict data in the later 13 days. Network parameters are consistent with setting method in this paper, namely BP network structure is 3-15-1. Based on the experiment, the absolute average prediction precision is $12.4750 \%$ and the maximum prediction precision is $29.6409 \%$.

2) Prediction of wavelet neural network: morlet wavelet functions are adopted in the hidden layer, sigmoid functions are used in the output layer and the network structure is 3-15-1, then the absolute average prediction precision is $10.9667 \%$ and the maximum prediction precision is $23.5421 \%$.

From the experiment above, the methods proposed in this paper have the characteristic of stable prediction result, which gets an advantage over single prediction result of BP and wavelet neural network, and BP and wavelet neural network require being predicted for many times to select more preferable values. The experiment also indicates that this data has a worse regularity and it is difficult to educe any ideal prediction result, most data on the spot have similar characteristics in fact.

\section{WAVELET DENOISING PREDICTION OF ORIGINAL DATA}

Because the original data contain interfering noises, wavelet denoising treatment is applicable: wavelet decomposition is applicable for original signals and most noise signals usually are included in the details with higher frequency so that decomposed wavelet coefficients are treated by finite threshold and so on, then wavelet reconstruction is performed for signals to reach the signal noise reduction purpose. Original data are decomposed into five layers by sym6 wavelet and the first layer of high-frequency coefficients are used to estimate the standard noise difference, soft thresholds and saved low-frequency signals are used for reducing signal noises. Denoising is performed by the following order:

$\mathrm{xd}=$ wdencmp('gbl',c,l,'sym6',5,thr,'s',keepapp) (5)

In the formula, wdencmp is a function which is used for removing and reducing noises of ID or 2D signals, "sym6" is the name of wavelet function, [c,l] indicates the structure of wavelet decomposition, wavelets are decomposed into 5 layers, thr indicates threshold vector, 's' indicates the selection of soft threshold, here keepapp $=1$ which indicates that no threshold quantization is available for low-frequency coefficients. After denoising, chaotic characteristic parameters $\tau$ and $m$ keep changeless, and Figure 6 indicates schematic diagram of equivalent attractors after wavelet denoising.

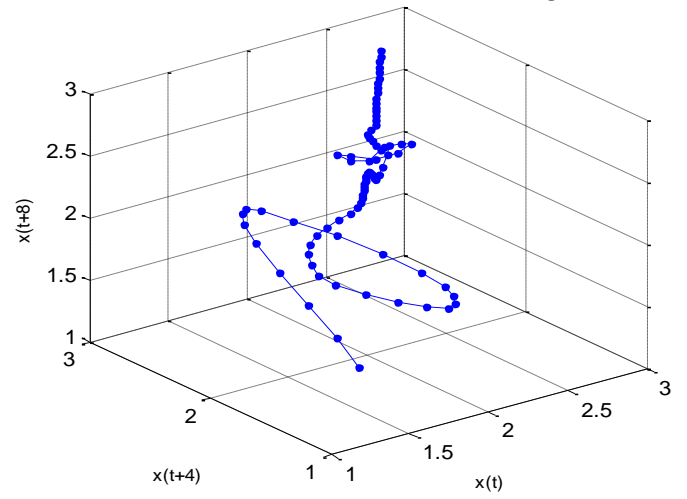

Fig. 6. Three-dimension equivalent chaotic attractor 


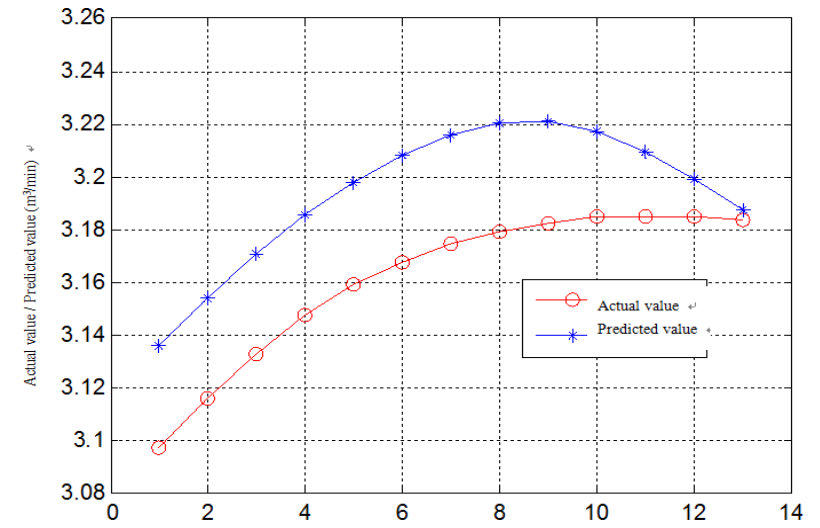

Fig. 7. Prediction result after signal denoising

The phase space is reconstructed in accordance with $\tau=4, m=5$, taking $\mathrm{Q}=14$. The prediction result is as follows: pre $=\left[\begin{array}{llllll}1.2289 & 1.2033 & 1.1916 & 1.1859 & 1.2155 & 1.2642\end{array}\right.$ $\begin{array}{llllll}1.2889 & 1.2781 & 1.1955 & 1.0041 & 0.7448 & 0.4520\end{array}$ $0.1230] \%$;

After wavelet denoising, the absolute average precision is $1.0289 \%$ and the maximum precision is $1.2889 \%$. Obviously, the prediction precision is improved greatly after signal denoising.

\section{CONCLUSION}

As for time series which has no chaotic characteristics or whose chaotic characteristics are fuzzy, state phase space is adopted to reconstruct modeling prediction, so that the time series prediction is transformed into an equivalent multiple regression prediction. Mackey-Glass time series prediction experiment shows that the delay factor and embedding dimension determined by traditional chaotic theory analysis are not always the optimal, phase spaces and neural network model parameters with good effect are selected through several calculations and optimization method is also adopted for optimization. As for type-I system with fuzzy chaotic characteristics such as gas emission time series, the popularization and prediction of phase space reconstruction theory are effective according to experiment. Simultaneously, a new method, which solves the randomness of neural network prediction result, is proposed in this paper to achieve a stable prediction result and accurately fit equivalent chaotic attractors. The research result in this paper provides a new approach for the analysis and prediction of complex nonlinear time series.

\section{REFERENCES}

[1] Hong-qing ZHU, Wen-jie CHANG, Bin ZHANG. Different-source gas emission prediction model of working face based on BP artificial neural network and its application. Journal of China Coal Society,2007,32(5) : 504-508. (In Chinese)

[2] Chuan-qu ZHU. Neural network model for predicting the amount of gas emitted from coal face. China Safety Science Journal, 1999, 9(2): 42-45. (In Chinese)

[3] Song GU, Zhen-wen ZHANG, Guo-jun LI. Forecasting Method of Grey Theory and Wavelet Neural Network for Mine Gas Gushing. Coal Technology, 2009, 28(10) : 123-125. (In Chinese)

[4] Min HAN. Chaotic time series prediction theory and method. Beijing: Water Conservancy and Electricity Press of China, 2007,5:79-81. (In Chinese)

[5] Xiang-cheng JIANG. Chaos property and prediction method of hydrological time series. Water Conservancy and Electricity Press of China, 2011,7:4-5.

[6] Wen-biao HUANG, Shi-liang SHI. Predicting on the time series of gas emission based on improved Lyapunov index. Journal of China Coal Society, 2009, 34(12) : 1665-1668. (In Chinese)

[7] Wei-wei HUANG, Qiang WANG, Shuang LU, Zhen-jiang LIU. To forecast the gas emission by neural network based on chaotic time series. Coal, 2005,14(5):7-9. (In Chinese)

[8] Jian CHENG, Jing-yi BAI, Jian-sheng QIAN, Shi-yin LI. Short-Term Forecasting Method of Coalmine Gas Concentration Based on Chaotic Time Series. Journal of China University of Mining \& Technology, 2008, 37(2) : 231-235. (In Chinese)

[9] Li GAO, Yan-jun HU, Hong-Zhen YU. Prediction of gas emission time series based on W-RBF. Journal of China Coal Society, 2008, 33(1) : 67-70. (In Chinese)

[10] Chun-bo XIU, Meng XU. Multi-step prediction method for time series based on chaotic operator network. ACTA PHYSICA SINICA, 2010, 59(11): 7650-7656. (In Chinese)

[11] Kai WANG, Qi-xiang YU. Non-linear characteristics and prediction model of Coal and gas outburst[M]. China University of Mining and Technology Press, 2005:20-21. (In Chinese)

[12] Jin-hu LV, An-jun CHEN, Shi-hua CHEN. Chaotic time series analysis and its application. Wuhan university press, 2005, 5: 57-59. (In Chinese)

[13] Chun-Tao ZHANG, Qian-Li MA, Hong PENG. Chaotic time series prediction based on information entropy optimized parameters of phase space reconstruction. ACTA PHYSICA SINICA, 2010,59(11):76237628. (In Chinese)

[14] ATAEI M, LOHMANN B, KHAKI-SEDIGH A, et al. Model based method for estimating an attractor dimension from uni/multivariate chaotic time series with application to Bremen climatic dynamics. Chaos, Solitons and Fractals, 2004, 19(5):1131-1139.

[15] Chang-sheng XIANG, Zhe-ming YUAN, Zi-ying ZHOU. Parameters Joint Optimization of Chaotic Time Series Prediction Model. Information and Control, 2011,40(5): 673-679. (In Chinese)

[16] Yong YOU, Wan-xing SHENG, Sun-an WANG. The Study and Application of the Electric Power System Short-term Load Forecasting Using a New Model. Proceedings of the CSEE, 2002, 29(9): 15-18. (In Chinese)

[17] Wenzhong JIANG, Zhong-gang HUO, Yu-jin QIN. Predicted technology of mine gas emission. COAL SCIENCE AND TECHNOLOGY, 2008, 36(6): 1-4. (In Chinese)

[18] Xue-zhi ZHAO, Chun-hua ZOU, Tong-jian CHEN, Bang-yan YE, Yong-hong PENG. A researeh on the Initialization of Parameters of Wavelet Neural Networks. Journal of South China University of Technology (Natural Scienee Edition), 2003,31(2): 77-79(In Chinese) 